\title{
Determinantes sociales de la salud y su asociación con el sobrepeso y obesidad en adolescentes
}

\author{
Social determinants of health and their association with adolescents being \\ overweight and obesity
}

\section{Determinantes sociais da saúde e sua associação com excesso de peso e obesidade em adolescentes}

\section{Paula A. Aristizábal-Vélez ${ }^{1}$ Diana A. García-Chamorro ${ }^{2}$, Consuelo Vélez-Alvarez ${ }^{3}$}

Tr, MSc, Docentes Universidad Autónoma de Manizales, Departamento de Salud Pública

Fisoterapeuta, Msc, Docente Universidad Autónoma de Manizales, Departamento de Salud Pública

Enfermera, PhD, Universidad Autónoma de Manizales - Antigua estación del Ferrocarril

Email:cva@ autonoma.edu.co.

\section{Resumen}

El propósito de este estudio es precisar la asociación entre los determinantes sociales de la salud, con el sobrepeso y la obesidad en adolescentes entre 15 y 19 años del municipio de Manizales. Es un estudio analítico de casos y controles, en 256 adolescentes (128 casos y 128 controles), residentes en el municipio de Manizales. Los grupos de investigación son personas entre 15 a 19 años con $I M C>=25 \mathrm{~kg} / \mathrm{m} 2$ (sobrepeso y obeso); el grupo control está compuesto por personas de 15 a 19 años de edad con IMC entre 18,5 y 24,99 Kg/m2. Fueron pareados por edad y género $1: 1$. El promedio de edad de los adolescentes fue de 17 años, $57,8 \%$ mujeres, promedio de IMC 24,27 kg/m2 +/-3,82 kg/m2. En relación a los determinantes sociales de la salud, no se encontró asociación entre el nivel de escolaridad, Seguridad Social en Salud o el estrato socioeconómico, y el sobrepeso y la obesidad. El nivel de escolaridad del tutor resultó ser un factor preventivo ante la presencia de obesidad en el adolescente OR 0,44 IC 95\% (0,21; $0,94) p=0,031$. Se concluye que el nivel de escolaridad del tutor del adolescente se asocia con el sobrepeso y la obesidad.

Palabras Clave: adolescente, factores epidemiológicos, obesidad, sobrepeso. (Fuente: DeCs Bireme)

\section{Abstract}

This study was aimed at determining the association between the social determinants of health for teenagers between 15 to 19 years old living in the town of Manizales with overweight and obesity. This was an analytical study of cases and controls, involving 256 adolescents (128 
cases and 128 controls) living in the town of Manizales. The young 15 to 19 year-old cases had a body mass index (BMI) >=25 kg/m2 (overweight and obese) and the young 15 to 19 year-old controls a 18.5 to $24.99 \mathrm{Kg} / \mathrm{m} 2 \mathrm{BMI}$. They were paired $1: 1$ by age and gender. The adolescents' average age was 17 years old, $57.8 \%$ were female and average BMI was $24.27 \mathrm{~kg} / \mathrm{m} 2$ +/-3.82 $\mathrm{kg} / \mathrm{m} 2$. Regarding the social determinants of health, no association was found between educational level, health-related social security and socioeconomic strata and being overweight and obesity. The legal representative's (parent/guardian) educational level proved to be a protective factor regarding obesity in adolescents (OR 0.44, 0.21-0.94 95\% $\mathrm{Cl}$; $\mathrm{p}=0.031$ ); it was thus concluded that adolescents' legal representatives' low educational level was associated with their being overweight and obesity.

Key words: adolescent, epidemiological factor, obesity, being overweight (source: DeCs Bireme).

\section{Resumo}

Determinar a associação entre determinantes sociais da saúde, com sobrepeso e obesidade em adolescentes de 15 aos 19 anos do município de Manizales. Estudo analítico de caso-controle com 256 adolescentes (128 casos e 128 controles) que moram na cidade de Manizales. Os casos de jovens com idade entre 15 a 19 anos com IMC> = 25 kg/m2 (sobrepeso e obesidade) e os controles com idade de 15 a 19 anos com um IMC entre 18,5 e 24,99 kg/m2. Foram pareados 01:01 por idade e sexo. A média de idade dos adolescentes foi de 17 anos, 57,8\% mulheres, com média de IMC 24,27 kg/m2 + / -3,82 kg/m2. Em relação aos determinantes sociais da saúde, não houve associação entre o nível de escolaridade, saúde previdência social e status socioeconômico eo excesso de peso e obesidade, o nível de escolaridade do representante legal acabou por ser um fator de proteção para a presença obesidade em adolescentes ou 0,44 IC 95\% $(0,21,0,94) p=0,031$. Conclui-se que o nível de escolaridade do representante legal do adolescente está associada ao excesso de peso e obesidade.

Palavras chave: adolescente, fatores epidemiológicos, obesidade, sobrepeso. (Fuente: DeCsBireme)

\section{Introducción}

La obesidad es un problema de salud pública tanto en los países desarrollados como en países en vías de desarrollo, con características epidémicas (Chescheir, 2011). En este sentido resulta muy preocupante cómo muchos adolescentes y jóvenes presentan sobrepeso y obesidad sin importar su condición social.

La Organización Mundial de la Salud (OMS) considera que la obesidad es una epidemia que inicia a edades tempranas con un origen multicausal low et al. (2009), y estima que en el 2015 se incrementará a 2,3 mil millones de personas con sobrepeso y 700 millones de personas obesas (Chescheir, 2011).

Según Low et al. (2009) en la actualidad se han encontrado estudios que muestran cómo el exceso de peso es un problema grave en los países desarrollados. En los países en desarrollo pasó de de $0,2 \%$ a $18,5 \%$ de la población, en los últimos años. De forma creciente, en los que están en vía de desarrollo, se estima un aumento de 0,1 a 35,3\%. El Centers for Disease Control and

Determinantes sociales de la salud y su asociación con el sobrepeso y obesidad en adolescentes 
Prevention de Estados Unidos (2010), reporta que, durante las últimas dos décadas, el porcentaje de adultos obesos ha aumentado más del doble y el $17 \%$ de los niños y adolescentes son obesos, con tendencia al aumento.

Según el trabajo realizado por Wang (2006), en América el 9,6\% de los niños en edad escolar tenían obesidad en el 2000 y se estimó que aumentaría a 15,2\% para el 2010. El sobre peso y la obesidad incrementan el riesgo de enfermedades crónicas como diabetes, hipertensión arterial, las enfermedades cardiovasculares, entre otras. Como consecuencia, la carga de enfermedad y mortalidad aumenta a largo plazo (Mispireta, et al., 2007 y Flegal, et al., 2007).

Para el caso de Colombia, la Encuesta Nacional de la Situación Nutricional (2010), muestra cómo la prevalencia de sobrepeso u obesidad entre la población de 5 a 17 años, ha aumentado un 25,9\% en el último quinquenio. Uno de cada 6 niños y adolescentes presenta sobrepeso u obesidad. Esta relación aumenta a medida que se incrementa el nivel del SISBEN y el nivel educativo de la madre $(9,4 \%$ en madres sin educación vs 26,8\% en madres con educación superior). El exceso de peso es mayor en el área urbana, con $19,2 \%$ vs el 13,4\% en el área rural. Los departamentos con mayores prevalencias de sobrepeso u obesidad son San Andrés 31,1\%, Guaviare 22,4\% y Cauca con $21,7 \%$.

Los trabajos desarrollados por Amador (2000) evidencian cómo la adolescencia es sumamente vulnerable a los factores ambientales, en particular los relacionados con la alimentación y la nutrición. A su vez, el comienzo y el ritmo del desarrollo sexual pueden influir en el consumo de alimentos. Los adolescentes que maduran más temprano suelen ser más gruesos; también suelen ser más gruesos los que tuvieron una reacción temprana de adiposidad en la niñez. Así mismo, la madurez temprana de los adolescentes está vinculada con un porcentaje mayor de grasa en el tronco.

El comportamiento alimentario del adolescente está influenciado por los hábitos familiares, la mayor vinculación social con sus pares y la creciente preocupación acerca de la imagen corporal, y por otro lado, por las necesidades de energía alimentaria. El curso de la obesidad desde la niñez hasta la edad adulta y el riesgo asociado a padecer enfermedades crónicas no transmisibles, destacan la importancia de las medidas preventivas durante la pubertad: a medida que una mayor cantidad de individuos se tornan obesos en edades tempranas, crece la repercusión de la obesidad como problema de salud pública. Esta condición, es uno de los trastornos nutricionales más comunes en la adolescencia y, a diferencia de otros trastornos que afectan la salud, tiene mayor consecuencia adversa en los planos individual, económico y social.

En el trabajo realizado por Fortich (2011) se resalta que existen determinantes sociales que podrían influenciar en el sobrepeso y la obesidad tales como la educación, el sexo, la pobreza, el lugar de residencia, entre otros.

Resulta relevante indagar sobre la relación de las determinantes sociales de la salud y el sobrepeso y la obesidad en la población entre 15 y 19 años de Manizales. Así se identifican los componentes físicos, emocionales y sociales que están generando una condición de salud inadecuada. Con los resultados se establecen prioridades para el diseño, implementación y evaluación de programas de prevención, atención y rehabilitación, a partir de los indicadores en salud. 


\section{Materiales y métodos}

Este fue un estudio analítico tipo casos y controles, que pretendió evidenciar la asociación existente entre los determinantes sociales de la salud (género, edad, afiliación a la seguridad Social en salud, nivel de escolaridad y estrato socioeconómico), y el sobrepeso y la obesidad en adolescentes.

Se tomó como población el total de adolescentes del Municipio de Manizales entre 15 y 19 años para el año 2010 que era de 32.028, distribuida en 16.229 hombres y 15.799 mujeres (Alcaldía de Manizales, 2010).

Para el cálculo de la muestra se tomó la información de la ENSIN 2010; dicho cálculo se hizo en el programa EPIDAT 3.1 (Programa para análisis epidemiológico de datos tabulados Organización Panamericana de la Salud - XUNTA DE GALICIA. CONSELLERIA DE SANIDADE. Dirección Xeral de Saúde Pública).

Los factores que intervinieron en el cálculo de la muestra son: proporción de casos expuestos o prevalencia de la exposición en los casos (p1); Proporción de controles expuestos o prevalencia de la exposición en los controles (p2); y Odds ratio esperado (OR). Estos tres valores están relacionados entre sí del modo siguiente:

$$
\mathrm{OR}=\begin{aligned}
& \mathrm{p} 1(1-\mathrm{p} 2) \\
& \mathrm{p} 2(1-\mathrm{p} 1)
\end{aligned}
$$

Proporción de casos expuestos: 26,80\% (obesos en madres con educación).

Proporción de controles expuestos: 9,40\% (obesos en madres sin educación).

OR esperado: 3,52.

Nivel de confianza: 95,0\%.
Potencia (\%) Número de pares

$\begin{array}{cc}80,0 & 79 \\ 85,0 & 90 \\ 90,0 & 104 \\ 95,0 & 128\end{array}$

Para el presente trabajo se asumió una potencia del 95\%, es decir se trabajó con 128 casos y 128 controles.

Casos: Jóvenes de 15 a 19 años de edad con IMC > = 25 hasta $29,99 \mathrm{~kg} / \mathrm{m} 2$ (sobrepeso).

Jóvenes de 15 a 19 años de edad con IMC >= $30 \mathrm{~kg} / \mathrm{m} 2$ (obeso).

Controles: Jóvenes de 15 a 19 años de edad con IMC entre 18,5 y $24,99 \mathrm{Kg} / \mathrm{m} 2$.

Los casos y controles fueron pareados por edad y género.

Fuente de los casos: fueron las escuelas, colegios y las universidades donde asisten en la actualidad los adolescentes de la Ciudad de Manizales.

Fuente de los Controles: fueron seleccionados de los mismos sitios de donde se hallaron los casos. Eso permitió controlar el sesgo de selección, ya que garantiza que compartan o compartieran un estilo de vida semejante.

Para seleccionar los sujetos de estudio se procedió a visitar los sitios definidos anteriormente. Se asumieron los siguientes criterios de inclusión:

1. Ser mayores de 15 años al momento de la evaluación y menores de 20 años.

2. Tener residencia y procedencia habitual en el municipio de Manizales. 
3. Ser clasificados en sobrepeso u obesidad en el momento de la valoración según el IMC para los casos. Para los controles, ser clasificados como normales según el IMC.

4. Aceptar su participación en el estudio mediante la firma del consentimiento informado.

5. Estar en plenas facultades mentales e intelectuales para diligenciar el cuestionario del estudio.

6. Para este estudio se tuvieron en cuenta los siguientes pasos:

1) Presentación del estudio.

2) Motivación para la participación de la población adolescente.

3) Reclutamiento de los participantes del estudio, según la muestra calculada.

4) Aceptación y firma del consentimiento informado.

5) Identificación de variables sociodemográficas.

6) Medición objetiva del peso y la talla utilizando balanza calibrada y cinta métrica y a partir de éstos el cálculo del IMC.

\section{Clasificación del índice de masa corporal (IMC).}

Health Organization (1997)

Categoría IMC (kg/m2)

Bajo peso: $<18,5$

Normal: 18,5-24,99

Sobrepeso: $>=25-29,99$

Obeso: $>=30$
7) Sistematización, tabulación y análisis de información. Para este procesamiento se utilizó el software estadístico SPSS versión 19, licenciada por la Universidad Autónoma de Manizales.

8) Análisis y discusión de resultados.

La técnica de recolección de información fue la encuesta. Se utilizó un cuestionario estructurado con el cual se indagó sobre datos sociodemográficos como edad, género, estudios realizados, máximos nivel de estudios del tutor, seguridad social, estrato socioeconómico, comuna. Se pesaron y tallaron y se calculo el IMC.

Para esta investigación se acogió la Resolución 008430 del Ministerio de Salud (1993). Este trabajo es considerado de riesgo mínimo, ya que no se realizó ninguna manipulación de variables de tipo biológico en los sujetos de estudio.

De acuerdo con la declaración de Helsinki (2000) esta investigación no debilita la resistencia física o mental del ser humano, y no se realizó experimentación terapéutica en seres humanos. En este sentido, la presente investigación tuvo un propósito eminentemente científico esto es, sin finalidad terapéutica con respecto a los pacientes. De acuerdo con lo estipulado por el Consejo de Organizaciones Internacionales de la Ciencias Médicas (1993), se contó con el consentimiento informado escrito de participación voluntaria en el estudio, y el asentimiento por parte de los menores de 18 años. El consentimiento informado, fue aprobado por el comité de ética de la Universidad Autónoma de Manizales.

\section{Resultados}

El $57,8 \%$ de los participantes fueron mujeres, el $42,2 \%$ hombres. La edad que predominó en los adolescentes encuestados fue de 17 años 
(26.6\%), el promedio de edad fue de 16,98 años +/- 1,32 años.

En relación al nivel educativo en el momento del estudio, se halló que el 52,3\% culminó los estudios primarios; el 46,1\%, estudios secundarios y el 1,6\% habían finalizado otro tipo de estudio (técnico ó tecnológico).

La distribución por comuna muestra que el mayor número de personas evaluadas residen en La Fuente, con un 18\%; seguido de Ciudadela del Norte $(17,6 \%)$; el menor número de personas residen en la comuna San José y el corregimiento Agroturístico $(0,4 \%)$. Es de anotar que en el estudio participaron personas de todas las comunas del municipio de Manizales (ver tabla 1).

Tabla 1.

Distribución de la muestra según el Género, Edad y Nivel de Escolaridad del encuestado. $(n=256)$

\begin{tabular}{|c|c|c|c|}
\hline \multicolumn{2}{|c|}{ Variables } & \multirow{2}{*}{$\begin{array}{c}\text { Frecuencia } \\
148 \\
\end{array}$} & \multirow{2}{*}{$\frac{\%}{57.8}$} \\
\hline Cómono & Mujeres & & \\
\hline Genero & Hombres & 108 & 42.2 \\
\hline \multirow{5}{*}{ Edad } & 15 & 44 & 17.2 \\
\hline & 16 & 52 & 20.3 \\
\hline & 17 & 68 & 26.6 \\
\hline & 18 & 50 & 19.5 \\
\hline & 19 & 42 & 16.4 \\
\hline \multirow{3}{*}{ Nivel Educativo } & estudios primarios & 134 & 52,3 \\
\hline & estudios secundarios & 118 & 46,1 \\
\hline & Otro & 4 & 1,6 \\
\hline
\end{tabular}

Fuente: elaboración propia.

En relación a los Determinantes sociales de la salud, se encontró que el nivel de escolaridad máxima del tutor (padre, madre o acudiente del adolescente) es universitario 35,5\%, seguido de estudios secundarios con el 35,2\%.

De los adolescentes evaluados, el 72,7\% pertenecían al régimen contributivo, mientras que el $16 \%$ se encontraban en el régimen subsidiado.

El estrato socioeconómico predominante fue 3 con un porcentaje del 44,9\%, seguido del estrato 2 con el 20,3\%. Cabe resaltar que en el estudio se encontró representación de todos los estratos socioeconómicos (ver tabla 2). 
Tabla 2.

Distribución de la muestra según los determinantes sociales de la Salud:

Escolaridad Máxima del Representante Legal, Afiliación a Seguridad

Social y Estrato Socioeconómico $(n=256)$

\begin{tabular}{|c|c|c|c|}
\hline Variables & & Frecuencia & $\%$ \\
\hline \multirow{5}{*}{ Escolaridad máxima del representante legal } & sin estudio & 3 & 1,2 \\
\hline & estudios primarios & 33 & 13 \\
\hline & estudios secundarios & 90 & 35 \\
\hline & estudios universitarios & 91 & 36 \\
\hline & otro & 39 & 15 \\
\hline \multirow{5}{*}{ Seguridad Social en Salud (del adolescente) } & contributivo & 186 & 73 \\
\hline & subsidiado & 41 & 16 \\
\hline & pobre no afiliado & 4 & 1,6 \\
\hline & régimen especial & 24 & 9,4 \\
\hline & Medicina Prepagada & 1 & 0,4 \\
\hline \multirow{6}{*}{ Estrato Socioeconómico } & Estrato 1 & 12 & 4,7 \\
\hline & Estrato 2 & 52 & 20 \\
\hline & Estrato 3 & 115 & 45 \\
\hline & Estrato 4 & 44 & 17 \\
\hline & Estrato 5 & 17 & 6,6 \\
\hline & Estrato 6 & 16 & 6,3 \\
\hline
\end{tabular}

Fuente: elaboración propia.

La distribución de la variable IMC mostró como parámetros de referencia que la media fue de 24,27 kg/m2, mínimo 18,2kg/m2 y máximo 32,40kg/m2 ـ 3,8283kg/m2 (ver Tabla 3).

Tabla 3.

Estadísticos descriptivos del IMC

\begin{tabular}{lccccc}
\hline & N & Mínimo & Máximo & Media & Desv. Típ. \\
\hline Índice de masa corporal & 256 & 18,2 & 32,4 & 24,27 & 3,8283 \\
\hline
\end{tabular}

Fuente: elaboración propia. 
La distribución del índice de masa corporal a partir del sobrepeso y la obesidad grado I, fue de $48,4 \%$ y $1,6 \%$, respectivamente (ver Tabla 4 ).

Tabla 4.

Clasificación del índice de masa corporal (IMC)

de la muestra participante $(\mathrm{n}=256)$

\begin{tabular}{|c|c|c|c|}
\hline & & Frecuencia & $\%$ \\
\hline \multirow{4}{*}{ IMC (kg/m²) } & Normopeso & 128 & 50 \\
\hline & Sobrepeso & 124 & 48,4 \\
\hline & Obeso grado I & 4 & 1,6 \\
\hline & Total & 256 & 100 \\
\hline
\end{tabular}

Para el análisis específico por casos y controles, se logró identificar que el nivel de escolaridad de los adolescentes tiene una representación de los casos en un 57\% en el nivel de secundaria; mientras que en los controles, el 52,3\% se encontró en un nivel universitario y otro (técnico y tecnológico). No se encontró asociación entre el nivel de escolaridad y la obesidad, OR 1,46 IC $95 \%(0,89 ; 2,38)$. Confirmado a través del X².

Existe asociación entre el nivel de escolaridad del tutor y la obesidad del adolescente. Esta asociación se confirmó con el valor de OR 0,44 IC 95\% $(0,21 ; 0,94)$ y el valor de $X^{2}$ ( $\left.p=0,031\right)$.

Respecto a la seguridad social en salud, se evidenció que no existe asociación entre el régimen de afiliación a la seguridad social en salud con la obesidad, dato confirmado con lo encontrado en el OR y el valor de $X^{2}(p=0,313)$.

En cuanto al estrato socioeconómico, se encontraron datos similares en los casos vs controles. El $30,5 \%$ de los y el $29,7 \%$ de los controles casos son de estrato alto. Los datos evidenciaron que no existe asociación entre dichas variables en los casos vs los controles, lo cual es confirmado con el valor de OR y chi cuadrado (Tabla 5). 
Tabla 5.

Determinantes sociales de la salud en los casos y controles

\begin{tabular}{|c|c|c|c|c|c|}
\hline Nivel de escolaridad & $\begin{array}{l}\text { Caso } \\
n=128\end{array}$ & $\begin{array}{c}\text { Control } \\
n=128 \\
\end{array}$ & OR & IC $95 \%$ & $\begin{array}{c}\text { Valor } \\
\text { p }\end{array}$ \\
\hline \multirow{2}{*}{ Secundaria } & 73 & 61 & \multirow{4}{*}{1,46} & \multirow{4}{*}{0,$89 ; 2,382,255$} & \multirow{4}{*}{0,133} \\
\hline & $57,00 \%$ & $47,60 \%$ & & & \\
\hline \multirow{2}{*}{ Universitario-otro } & 55 & 67 & & & \\
\hline & $43,00 \%$ & $52,30 \%$ & & & \\
\hline \multicolumn{6}{|c|}{ Nivel de escolaridad de representante } \\
\hline \multirow{2}{*}{ Básico } & 12 & 24 & \multirow{4}{*}{0,44} & \multirow{4}{*}{0,$21 ; 0,944,655$} & \multirow{4}{*}{0,031} \\
\hline & $9,40 \%$ & $18,80 \%$ & & & \\
\hline \multirow{2}{*}{ Superior } & 116 & 104 & & & \\
\hline & $90,60 \%$ & $81,20 \%$ & & & \\
\hline \multicolumn{6}{|c|}{ Seguridad social en salud } \\
\hline \multirow{2}{*}{ Régimen contributivo } & 1 & 3 & \multirow{4}{*}{0,33} & \multirow{4}{*}{0,$03 ; 3,191,016$} & \multirow{4}{*}{0,313} \\
\hline & $0,80 \%$ & $2,30 \%$ & & & \\
\hline \multirow{2}{*}{ Régimen subsidiado } & 127 & 125 & & & \\
\hline & $99,20 \%$ & $97,70 \%$ & & & \\
\hline \multicolumn{6}{|l|}{ Estrato socioeconómico } \\
\hline Bajo-medio & $\begin{array}{c}89 \\
6950 \%\end{array}$ & $\begin{array}{c}90 \\
70 \%\end{array}$ & \multirow{3}{*}{0,96} & \multirow{3}{*}{0,$56 ; 1,640,019$} & \multirow{3}{*}{0,892} \\
\hline \multirow{2}{*}{ Alto } & $\frac{69,50 \%}{39}$ & & & & \\
\hline & $30,50 \%$ & $29,70 \%$ & & & \\
\hline
\end{tabular}

Fuente: elaboración propia.

\section{Discusión}

La perspectiva canadiense plantea la influencia de los determinantes sociales de la salud sobre la obesidad en los adolescentes, la cual se podría explicar por los siguientes factores: ingreso y nivel social, redes de apoyo social, educación, empleo y condición de trabajo, entornos sociales, entornos físicos, prácticas de salud personales y aptitudes de adaptación, características biológicas y genéticas, servicios de salud, género y cultura. (Health Canada, 2002).

Los resultados sociodemográficos, están acordes con los publicados por Sánchez et al. (2013), quienes reportan que la prevalencia del exceso de peso es superior en niños que en niñas y muy superior en el grupo de 8 a 13 años, y se asocia con la clase social y el nivel de escolaridad, convirtiendo ésta situación en un problema de salud pública a nivel mundial. Lo anterior, genera a su vez graves trastornos metabólicos que Ilevan al aumento en los índices de mortalidad Barquera et al., (2013) y Felix, Redondo et al. (2012).

Según Redondo et al. (2012), diversos estudios han encontrado complicaciones relacionadas con la obesidad, como las enfermedades cardiovasculares (hipertensión arterial a expensa de la sistólica y diastólica, isquemias coronarias) y metabólicas (dislipidemia, gota, diabetes tipo II). Además resaltan que dichas complicaciones están íntimamente 
relacionadas con el grado, la localización (obesidad androide) y el tiempo de evolución del sobrepeso.

El incremento paralelo de la frecuencia de la obesidad y del síndrome metabólico es un fenómeno mundial, situación que García et al. (2008), Oquendo et al. (2010), Lama et al. (2006) y Poletti. (2007) han documentado de manera simultánea en la población infantil y adolescente; situación que, a futuro, podría generar problemas de comorbilidad asociada en los adolescentes participantes en este estudio, así como producir un aumento significativo del riesgo de enfermedades crónicas.

Algunas de la variables sociodemográficas analizadas están relacionadas con la edad y género. En investigaciones similares, se encontró que la edad de mayor representatividad fue de 14 años, con participación de mujeres en un 59\%. El peso corporal presentó un promedio de $97,1+/-24.9 \mathrm{~kg}$ y la talla $1,64 \mathrm{mt}+/-9,6$ (ENSIN, 2010). En el presente estudio, el promedio de edad de los adolescentes fue de 17 años; el género que predominó fue el femenino, en un $57,8 \%$; el peso corporal, $64,183 \mathrm{~kg}+/-8,897 \mathrm{~kg}$; y la talla, 1,62 mt $+/-0.069 \mathrm{mt}$.

Según Álvarez et al. (2012), investigaciones internacionales demuestran cómo el sobrepeso y la obesidad pueden presentarse en los adolescentes sin que exista una relación directa con su condición social. Esto se evidencia en este estudio, en el que el estrato socioeconómico no mostró relación con la presencia de sobrepeso y obesidad. No obstante, Cano et al. (2010) relacionan el sobrepeso y la obesidad en los adolescentes como un marcador de desigualdad en salud.

Los estudios nacionales respecto a los determinantes sociales de la salud, muestran cómo a mayor nivel educativo y estrato socioeconómico del tutor, se genera mayor riesgo para la presencia del sobrepeso y la obesidad en los adolescentes (ENSIN, 2010). El anterior dato difiere de los resultados de esta investigación, en la cual se encontró que a mayor nivel educativo de la madre menor proporción de sobrepeso y obesidad.

Frente al estrato socioeconómico, en congruencia con el estudio de Tuesca et al. (2008), se encontró que la mayoría de encuestados pertenecen al estrato 3 y 4 , dato que coincide con esta investigación, en la que se encontró que el mayor número de participantes pertenecían al estrato 3 .

\section{Conclusiones}

Entre los participantes del estudio se presentó una mayor frecuencia de sobrepeso y obesidad en las mujeres. La edad promedio entre hombres y mujeres fue de 16,98 años.

Frente al comportamiento de los determinantes sociales de la salud, tanto para el grupo de casos como para los controles, se encontró que la mayoría de los participantes alcanzó a culminar sus estudios primarios; el máximo nivel de escolaridad del tutor fue universitario; los adolescentes se encuentran afiliados a la seguridad social en salud y el mayor porcentaje de los adolescentes viven en estrato socioeconómico 3.

El nivel educativo del tutor se asocia con el sobrepeso y la obesidad de los adolescentes participantes en el estudio. A mayor nivel de escolaridad del cuidador del adolescente, menor riesgo de que esté presente sobrepeso u obesidad.

Aunque determinantes sociales como la edad, género, escolaridad, estrato socioeconómico no mostraron asociación 
estadísticamente significativa con el sobrepeso y la obesidad en los adolescentes estudiados, ambos problemas tienen una distribución en forma de gradiente en los adolescentes reflejando una desigualdad social.

\section{Recomendaciones}

Involucrar en los programas escolares un abordaje integral en los adolescentes, desde acciones de promoción y prevención orientadas al logro del autocuidado de la salud con la participación activa del padre, madre o cuidador.

Establecer acciones o programas que permitan identificar tempranamente desordenes alimentarios que generan situaciones de riesgo y puedan llevar al sobrepeso y la obesidad en este grupo poblacional.

Teniendo en cuenta que el sobrepeso y la obesidad son factores de riesgo para la presencia de patologías crónicas no transmisibles en toda la población, es necesario retomar los resultados de este estudio para identificar y orientar políticas públicas que se operativicen en acciones para minimizar éstos efectos, mejorar las condiciones de salud y disminuir las brechas sociales.

Realizar otras investigaciones que permitan evidenciar la asociación entre otros factores y el sobrepeso y la obesidad del adolescente, como por ejemplo el uso del tiempo libre.

\section{Referencias}

Alcaldía de Manizales, Secretaría de Salud Pública Perfil epidemiológico Manizales 2009-2010. Aspectos demográficos.
Álvarez D, Sánchez J, Gómez G, Tarqui C. Sobrepeso y Obesidad: Prevalencia y Determinantes Sociales el exceso de peso en la Población Peruana (2009- 2010). Rev Peru Med Exp Salud Pública. 2012; 29(3):303-13.

Amador M. La obesidad en la adolescencia. En: Peña M, Bacallao, editor. La obesidad en la pobreza. OPS/OMS Publicación Científica 576. 2000:125-13 [en línea]. p. 125-126. [consultado 2010 Octubre 5]. Disponible en: http://www.bibliomed. com/biblioteca/paho/obesidad/4b.pdf

Barquera S, Campos I, Hernández L, Pedroza A, Rivera JA. Prevalencia de obesidad en adultos mexicanos, 2000-2012. Salud pública Méx [revista en la Internet]. 2013 [citado 2014 Sep 23] ; 55( Suppl 2 ): S 151 - S 160 . Disponible en : http://www.scielo.org.mx/scielo.php?scrip $\mathrm{t}=\mathrm{sci} \_$arttext $\&$ pid $=$ S00363634201300080 $0012 \& \operatorname{lng}=$ es

Cano A, Alberola S, Casares I, Pérez I. Desigualdades sociales en la prevalencia de sobrepeso y obesidad en adolescentes. An Pediatr. 2010;73(5):241-8.

Centers for Disease Control and Prevention (2010) Obesity_At a Glance, 2007

Consejo de Organizaciones Internacionales de las Ciencias Médicas (CIOMS), OMS. Pautas Éticas Internacionales para la Investigación y Experimentación Biomédica en Seres Humanos, Ginebra. 1993. p. 53.

Chescheir N. Obesidad en el Mundo y su Efecto en la Salud de la Mujer. Obstet Gynecol. 2011;117:1213-22.

Encuesta Nacional de la Situación Nutricional en Colombia 2010. Resumen Ejecutivo 
ENSIN 2010 [en línea]. [consultado 2011 Marzo 16]. Disponible en:http://www. bogotamasactiva.gov.co/files/Resumen\%2 OEjecutivo\%20ENSIN\%202010.pdf

Félix-Redondo FJ, et al. Prevalencia de obesidad y riesgo cardiovascular asociado en la población general de un área de salud de Extremadura. Estudio Hermex. Endocrinol Nutr. 2012. doi:10.1016/ j.endonu.2012.01.005.

Flegal K, Barry G, David W, Mitchell G. Cause-specific excess deaths associated with underweight, overweight, and obesity. JAMA. 2007; 298(17):2028-37.

Fortich R, Gutiérrez J. Los determinantes de la obesidad en Colombia.Economía \& Región. 2011;5(2):155-82.

García E, De la Llata M, Kaufer M, Tusié MT, Calzada R, Vázquez V, et al. La obesidad y el síndrome metabólico como problema de salud pública: una reflexión. Salud pública Méx [serial on the Internet]. 2008 Dec [cited 2014 Sep 23] ; 50(6): 530-547.

Health Canada. The Social Determinants of Health: an overview of the implications for policy and the role of the health sector. Ottawa: York University; 2002.

Lama RA, Franch A, Gil CM. Obesidad Infantil. Recomendaciones del Comité de Nutrición de la Asociación Española de Pediatría. Parte I. Prevención. Detección precoz. Papel del pediatra. Anales de Pediatría. 2006;65:606-615 [en línea]. [consultado 2011 Enero 31]. Disponible en:http://db.doyma.es/ cgibin/wdbcgi.exe/doyma/mrevista.fulltext ?pident $=1305854$.

Low S, Chin M, Deurenberg-Yap M. Review on Epidemic of Obesity. Ann Acad Med Singapore. 2009; 38:57-65.
Mispireta M, Rosas A, Velásquez J, Lescano A, Lanata C. Transición Nutricional en el Perú, 1991 - 2005. Rev Peru Med Exp Salud Pública. 2007; 24(2):129-35.

Oquendo Y, Piñeiro R, Duarte MC, Guillen A. Síndrome metabólico en niños y adolescentes hipertensos obesos. Rev Cubana Pediatr [revista en la Internet]. 2010 Dic [citado 2014 Sep 23]; 82(4): 31-40. Disponible en: http://scielo.sld.cu/ scielo. php? script=sci_arttext \&pid $=$ S00347 $5312010000400004 \& \operatorname{lng}=$ es.

Poletti OH, Barrios L. Obesidad e Hipertensión arterial en escolares de la ciudad de Corrientes: Argentina. Arch Argent Pediatr. 2007; 105(4):293-298.

República de Colombia. Ministerio de Salud. Resolución No. 008430. Santafé de Bogotá; 1993.

Sánchez JJ, Jiménez JJ, Fernández F, María J. Sánchezac MJ. Prevalencia de obesidad infantil y juvenil en España en 2012. Rev Esp Cardiol. 2013; 66(5): 371-6.

Tuesca R, Centeno. RH, Ossa SM, García DN, Lobo LJ. Calidad de vida relacionada con la salud y determinantes sociodemográficos en adolescentes de Barranquilla (Colombia). Salud Uninorte. 2008; 24(1):53-63.

Wang Y, Lobstein T. Worldwide trends in childhood overweight and obesity. Int J Pediatr Obes. 2006; 1(1):11-25.

WHO. Obesity: Preventing and managing the global epidemic. Ginebra. World Health Organization, June 3-5, 1997. (WHO technical report series No. 894). 